\title{
The absorption-peak map of Mare Serenitatis obtained by a hyper-spectral telescope
}

\author{
Hideaki Okuno, Yuta Yamanoi, and Kazuto Saiki \\ Department of Earth and Space Science, Graduate School of Science, Osaka University, Osaka 560-0043, Japan
}

(Received February 2, 2007; Revised October 5, 2007; Accepted November 22, 2007; Online published April 9, 2008)

\begin{abstract}
The $\mathrm{Mg}$-number $[\mathrm{Mg \#}=$ atomic $\mathrm{Mg} /(\mathrm{Mg}+\mathrm{Fe})]$ serves as an important petrologic discriminator when analyzing and understanding lunar rocks. Variations in the $\mathrm{Mg \#}$ shift the wavelength of the absorption spectra of ferrous iron, which peak at around $1000 \mathrm{~nm}$. Based on the image cubes of the Moon obtained by the Advanced Lunar Imaging Spectrometer (ALIS), we detected the shift in the absorption spectra of ferrous iron and built up an absorption-peak map of Mare Serenitatis. The wavelength of the absorption peak shows an 11-nm shift in Mare Serenitatis. Since the degree of space weathering can be considered to be almost the same as that within the same lava unit and $\mathrm{Ca}$ content cannot change without changing Mg\# during magma differentiation, these shifts of the peak absorption spectra suggest that there is $\mathrm{Mg \#}$ variation in at least the same lava unit.
\end{abstract}

Key words: Moon, hyper-spectral telescope, Mg-number.

\section{Introduction}

Results from the 1994 Clementine mission (e.g., Nozette et al., 1994) and the 1998 Lunar Prospector mission (e.g., Blinder, 1998) have led to significant new views of the structure and distribution of materials in the crust and upper mantle of the Moon. In particular, Lucey et al. (1995, 1998, 2000), who developed a method for mapping iron and titanium abundance using Clementine ultraviolet-visible light (UV-VIS) data, greatly advanced the field of lunar science. The next step for understanding the origin and thermal evolution of the Moon is to construct a Mg-number [Mg\# = atomic $\mathrm{Mg} /(\mathrm{Mg}+\mathrm{Fe})]$ map of the pyroxene found on the lunar surface. For example, using $\mathrm{Mg \#}$ and the Fe-Mg partition coefficient between melt and mafic minerals reported by Stolper (1977), it is possible to estimate the degree of partial melting of source rocks before lava eruption. Variations in the Mg\# of the pyroxene shifts the wavelength of the absorption spectra of ferrous iron, which peak at around $1000 \mathrm{~nm}$ and $2000 \mathrm{~nm}$ (e.g., Hazen et al., 1978). Consequently, if $\mathrm{Mg \#}$ variations do actually exist in the materials comprising the lunar surface, it should be possible to detect the shift in the absorption peak. However, such shifting could not be detected by remote sensing because the latter is limited to a very small spectral range. Pieters and Englert (1993) reported that they had observed small variations between spectra for different mare craters at visible wavelengths as well as near the $1000 \mathrm{~nm}$ absorption peak using an Earth-based telescope and the center of absorption spectra ranges $980-1000 \mathrm{~nm}$ in the lunar mare. However, these authors did not describe the shifts caused by Mg\# variations in the lunar mare.

Copyright (c) The Society of Geomagnetism and Earth, Planetary and Space Sciences (SGEPSS); The Seismological Society of Japan; The Volcanological Society of Japan; The Geodetic Society of Japan; The Japanese Society for Planetary Sciences; TERRAPUB.
In this paper, we report our observations of the Moon using a hyper-spectral telescope named ALIS (Advanced Lunar Imaging Spectrometer) and our successful attempts to detect the absorption-peak shift of lunar mare basalts. We discuss the accuracy and the interpretation of the absorption-peak map that was obtained and estimate $\mathrm{Mg \#}$ distribution from the map.

\section{Method}

The spectral data of the lunar surface were obtained by ALIS (Saiki et al., 2004). ALIS is a hyper-spectral imaging telescope developed by one of the authors (KS) in collaboration with JFE Techno-Research Co. (Japan). The specifications of ALIS are shown in Table 1. ALIS is a cassegrain telescope equipped with two spectrometers-a VIS and a near-infrared (NIR) spectrometer-and a monitoring camera. Each spectrometer consists of an imaging sensor and a Prism-Grating-Prism (PGP) unit (ImSpector; manufactured by Specim Corp.). Incoming light passes through the opening in the primary mirror of the Cassegrain telescope to focus on a slit plane of the PGP unit. The PGP unit produces a "1-line spatial information" $\times$ "spectral information" image on the charged-coupled device (CCD) plane as one shot. The coverage of wavelength is 380-1100 nm (VIS) and 1000-1700 nm (NIR). Line images are assembled by scanning an image on the slit of the spectrometer by rotating a deflecting mirror. The mirror is also used to switch between the VIS-system, NIR-system, and the monitor camera. The monitor camera is used for adjusting the focus and frames. Spatial resolution of ALIS-VIS is $6.188^{\prime \prime} /$ pixel and that of ALIS-NIR is $6.435^{\prime \prime} /$ pixel. The NIR data were not used because the quality was not enough for this study due to the high level of dark noise. The typical scanning speed of the ALIS-VIS camera is about $3.2 \mathrm{~s}$ per 1 line, and the exposure time of each line is $50 \mathrm{~ms}$. One lunar image cube is composed of 512 scanning lines, which can be obtained in 
Table 1. Specification of ALIS (Advanced Lunar Imaging Spectrometer).

\begin{tabular}{|c|c|}
\hline Telescope type: & Cassegrain \\
\hline Aperture: & $200 \mathrm{~mm}$ \\
\hline Focal distance: & $800 \mathrm{~mm}$ \\
\hline Spectrometer type: & Prism-Grating-Prism \\
\hline Image scanning: & Mirror rotation system \\
\hline Spectrometer range: & NIR: $1000-1700 \mathrm{~nm}$ \\
\hline Wavelength resolution: & $9 \mathrm{~nm}(1000-1700 \mathrm{~nm})$ \\
\hline Sensor: & $\begin{array}{l}\text { VIS: Spectra video SV } 512 \text { (Pixel vision) } 512 \times 512 \text { pixels } \\
\text { NIR: SU320MS-1.7 RT (Sensors unlimited) } 320 \times 256 \text { pixels }\end{array}$ \\
\hline
\end{tabular}



Fig. 1. $700 \mathrm{~nm}$ reflectance image of Mare Serenitatis. Geological boundaries are based on Kodama and Yamaguchi (2003). Points MS A and MS B indicate the location where two sample spectra were obtained. The line defined by two points, from the Plinius crater (Lat: $15.4^{\circ} \mathrm{N}$, Long: $23.7^{\circ} \mathrm{E}$ ) to the Santos-Dumont crater (Lat: $27.7^{\circ} \mathrm{N}$, Long: $4.8^{\circ} \mathrm{E}$ ), indicates the sampling line for making Fig. 8.

about $28 \mathrm{~min}$. The scanning direction is vertical for the images used this study.

The location of the observation was Science City, located at the peak of Mt. Haleakala, Maui, Hawaii, USA. The observation dates were from 15 to 26 August 2005 and from 12 to 18 December 2005. The observations were carried out by the ALIS-users' group, which includes the authors. ALIS and its peripheral devices were transported from Japan. An observation dome, which had been constructed by Okano Laboratories at Tohoku University, Japan, and the observation support facilities were provided by the Mees Solar Observatory at the University of Hawaii.

Mare Serenitatis was selected as a survey area because it is located almost at the center of lunar near-side and the range of observation geometry is not so wide among the mare. Mare Serenitatis is located at $27^{\circ} \mathrm{N}, 19^{\circ} \mathrm{E}$ and is $740 \mathrm{~km}$ in diameter. A large part of the basin is filled with mare basalt. Most of the mare samples returned by Apollo 17 showed a Ti-rich composition, with their radioactive ages ranging from 3.56 to $3.79 \mathrm{Ga}$ (e.g., Schaeffer and Schaeffer, 1977; Tera et al., 1974). The stratigraphy of Mare Serenitatis has been well studied by many researchers (e.g., Carr, 1966; Howard et al., 1973). Based on previous results, the mare basalt in the center of the basin tends to be younger than that found at the margins of the mare. However, Wilhelms and McCauley (1971) and Boyce (1976) reported that several patches of mare basalt around the edge of the basin were younger than the central basalts. Pieters (1978) classified Serenitatis deposits into five groups based on four parameters: UV/VIS ratio, albedo, and $1000 \mathrm{~nm}$ and $2000 \mathrm{~nm}$ band strength. To enable more detailed descriptions, Kodama and Yamaguchi (2003) reclassified the Mare Serenitatis deposits into seven groups-Sr1, Sr2, Sr3A, $\mathrm{Sr} 3 \mathrm{~B}, \mathrm{Sr} 4, \mathrm{Sr} 5$, and DMM (Fig. 1) - using such parameters as $750 \mathrm{~nm}$ albedo, UV/VIS ratio, $1000 \mathrm{~nm}$ absorption signature, and $\mathrm{FeO}$ and $\mathrm{TiO}_{2}$ contents, which were based on a spectral analysis derived from Clementine UV-VIS multispectral data. The Sr2 unit is not shown in Fig. 1 because the shape of the $\mathrm{Sr} 2$ area is narrow and complicated. The areas of mISP, hDWA, and HDWA of Pieters' classification were subdivided into two areas ( $\mathrm{Sr} 4$ and $\mathrm{Sr} 3 \mathrm{~A}$ ) three areas (Sr1, Sr2, and Sr5), and two areas (Sr1 and Sr2), respectively, in Kodamas' classification.

\section{Image Processing}

To demonstrate our image processing, we processed two sample spectra. The locations (MS A and MS B) where the spectra were obtained are shown in Fig. 1, and the raw spectra are shown in Fig. 2(a). The spectral "dent" found at around $755 \mathrm{~nm}$ is caused by the edge of a low-cut filter covering the 750-1100-nm area of ALIS's grating. Data processing is required for obtaining a characteristic shape of lunar material because the shapes of these spectra are mainly formed by the sensitivity of the ALIS detector. In order to detect any slight shift in the absorption peak, we processed the image cube of the Moon as follows. (1) Dark and flat field correction was carried out (Fig. 2(b)). Dark field correction reduces the amount of dark current accumulating in the pixels during an exposure and data transfer and pixel-specific offsets in the CCD and camera electronics. Flat field correction reduces the effect of any sensitivitydifference between each pixel and the artificial shade induced by dust and optical vignetting. The flat field image cube was taken using an integrating sphere at JAXA. (2) Smoothing with a $20-\mathrm{nm}$ moving average was carried out for each pixel (Fig. 2(c)) to reduce random noise and to make the position of absorption band clear. (3) Normalization of all mare spectra with the same standard highland spectrum was carried out to reduce the influence of atmospheric absorption and the difference of relative sensitivity of CCD pixels at each wavelength (Fig. 2(d)). The standard 



Fig. 2. Example spectra showing process flow. (a) Raw spectra of two small areas MS A and MS B of Mare Serenitatis. (b) Corrected spectra after dark and flat correction (step 1 in the text). (c) Smoothed spectra after wavelength smoothing (step 2). (d) Spectra normalized by one highland standard spectrum (step 3). (e) Smoothed spectra after wavelength smoothing (step 4). Dashed line indicates a baseline for MS A. (f) Resultant spectra after baseline correction (step 5). Triangles indicate minimum positions of each spectrum. The peak positions of MS A and MS B are $978 \mathrm{~nm}$ and $967 \mathrm{~nm}$, respectively.

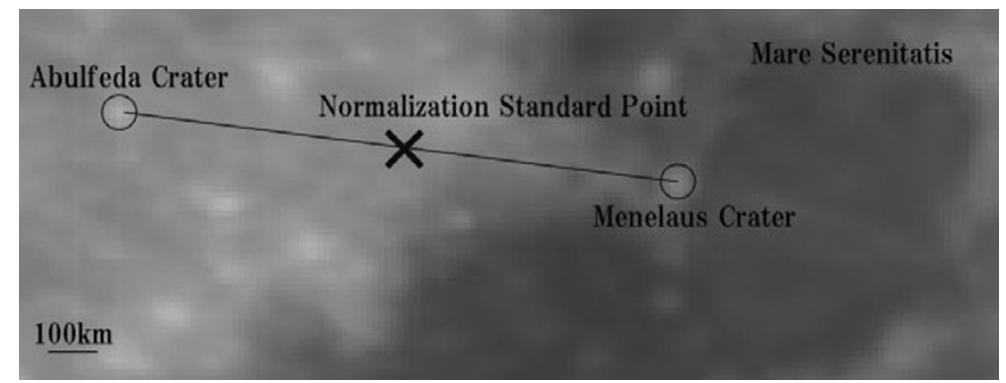

Fig. 3. The location of the standard highland. The point is defined at the center of Abulfeda crater and Menelaus crater.

point for the normalization is selected from the highland spectra because these have a small absorption peaks of ferrous iron (e.g., Lucey et al., 1995). To pick up the same standard point from different lunar images, we defined the point at the center of two conspicuous craters, Abulfeda and Menelaus (Fig. 3). Although normalized reflectance spectra cannot provide accurate information on the peak positions of the absorption band, the peak positions can be compared relatively to facilitate any discussion of differences in local geology. (4) Smoothing with a 20 -nm moving average was carried out again for each pixel (Fig. 2(e)). (5) A baseline correction was carried out to reduce the influence of lunar space weathering. Space weathering makes the reflectance spectra of lunar surface darker and redder and reduces the depth of the absorption band (e.g., Sasaki et al., 2001). Pieters and Englert (1993) defined a baseline of lunar re- 


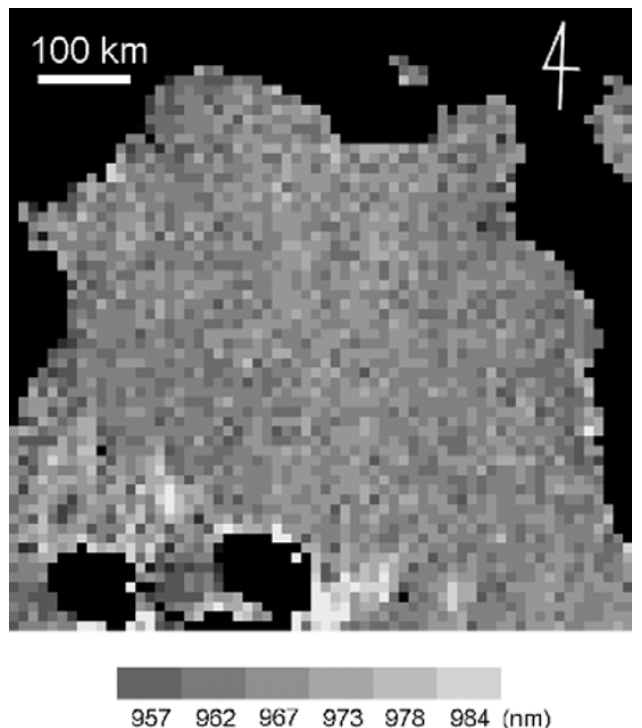

Fig. 4. The absorption peak map of Mare Serenitatis before spatial smoothing.

flectance spectra as a common tangent around $750 \mathrm{~nm}$ and $1500 \mathrm{~nm}$ and the spectra were divided by their own baseline. However, the same correction cannot be carried out for our data because visible system of ALIS covers only spectra from 380 to $1100 \mathrm{~nm}$ and our spectra are not reflectance but those normalized by highland spectra. Therefore, we defined a virtual baseline which starts at $693 \mathrm{~nm}$ and ends at $1059 \mathrm{~nm}$ and tried to reduce the influence of space weathering as much as possible by subtracting the baseline from each spectrum (Fig. 2(f)). Comparing the resultant spectra, we are able to detect the slight shifts in the absorption spectra (Fig. 2(f)). Minimum positions of spectrum MS A and MS B are $978 \mathrm{~nm}$ and $967 \mathrm{~nm}$, respectively. These processes were applied to all pixels on the lunar images, and maps of the wavelength of the absorption peak were then made (Fig. 4). (6) To reduce the effect of random error and obtain a distinct spatial pattern of the positions of the absorption peaks, we performed $3 \times 3$-pixel spatial smoothing (Fig. 5).

\section{Results}

The peak-position distributions of Mare Serenitatis are shown in Fig. 5, which ranges from 957 to $984 \mathrm{~nm}$. Figure 5(a) and (c) are obtained from data on 15 December, (b) from data on 14 December, (d) and (e) from data on 18 August, and (f) from data on 16 August. These six lunar observations were selected for further analysis because the weather was fine and the Moon was nearly full Moon on those nights. Our method of detecting the absorption-peak was not applied to highland areas because all spectra are normalized by one of the highland spectra, each of which has a weak absorption of ferrous iron. The absorption peak of highland pyroxene would not be detected with sufficient accuracy, therefore highland areas around Mare Serenitatis are colored in black (Fig. 5) and these areas are not discussed here. Although these six maps are slightly different from each other because of the effect of random error, the
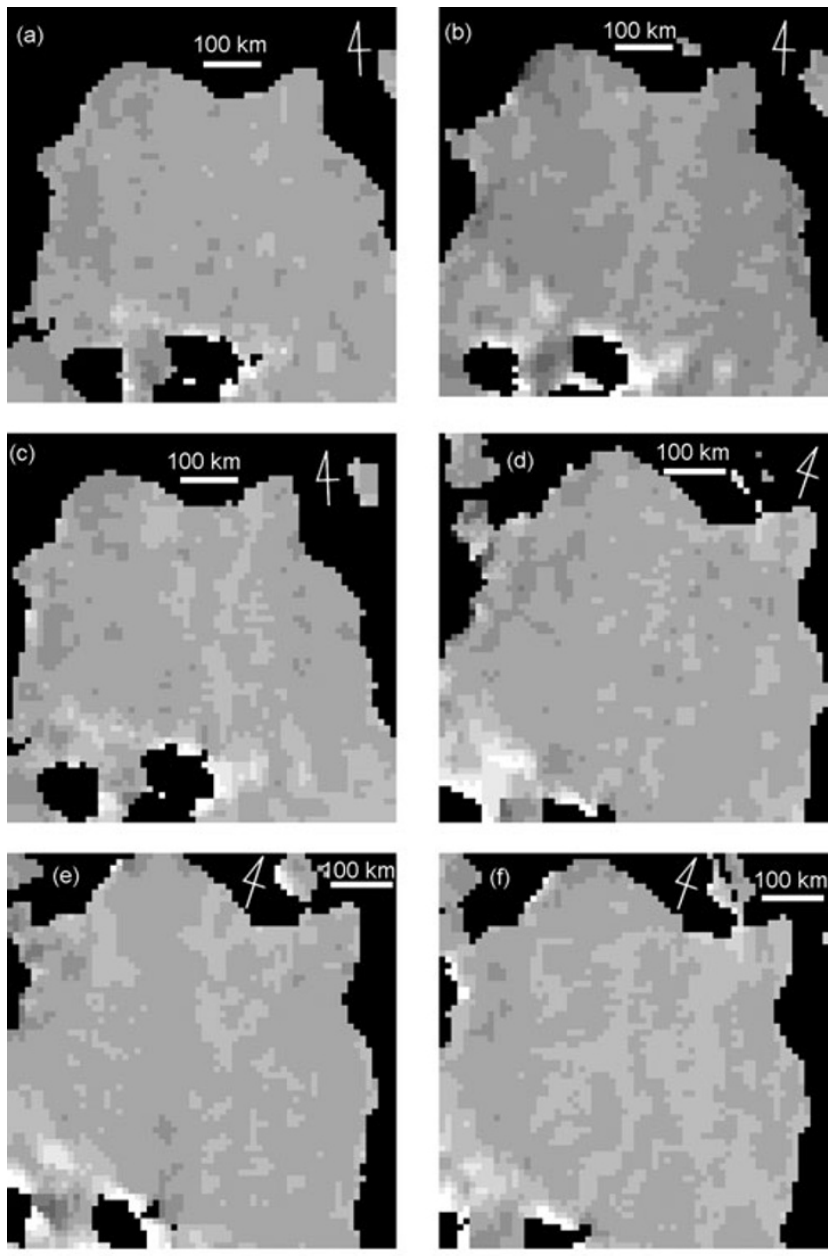

$957 \quad 962 \quad 967 \quad 973 \quad 978 \quad 984(n m)$

Fig. 5. The absorption peak maps of Mare Serenitatis. Images (a) and (c) are obtained on 15 December, (b) on 14 December, (d) and (e) on 18 August, and (f) on 16 August. For highland areas around Mare Serenitatis, the peaks are not defined and the areas are painted with black.

change in the optical depth of the atmosphere during the scan, remaining noise, among other factors, it is possible to observe similar spatial patterns when the six sets of data are compared. In order to discuss the characteristics of local area, we named these as given in Fig. 6. The peak positions of areas 1,2,3, and 4 are shorter than those of their surroundings. The peak positions of area 5 are longer than those of their surroundings and the shape of the areas are unstable. A primary cause of this ambiguous shape is most likely the reduction of the depth of the absorption band due to the ray materials from the Tycho crater.

There is a possibility that the incomplete baseline correction produces pseudo-peak shifts. To check the effect of baseline correction on the position of the absorption peak, we constructed a plot of the slope versus the peak position of all the pixels in Fig. 5(b) (Fig. 7). The correlation coefficient between the slope and the wavelength of absorption peak is -0.20 , and there is no systematic correlation between them. To compare the six images quantitatively, we plotted a profile of the average peak positions of the six images (Fig. 8) along the line defined by two points; Plinius 


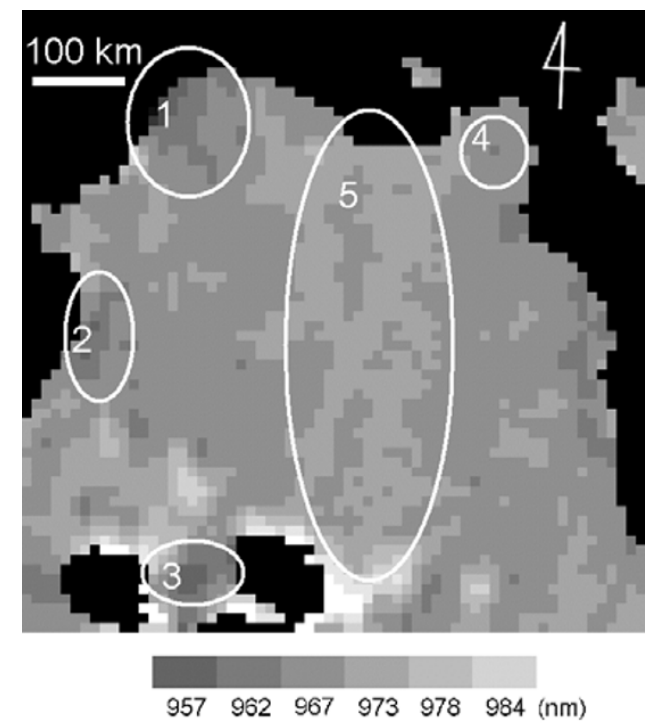

Fig. 6. The absorption peak map of Mare Serenitatis with indicators showing notable areas. The base image was obtained on 14 December.

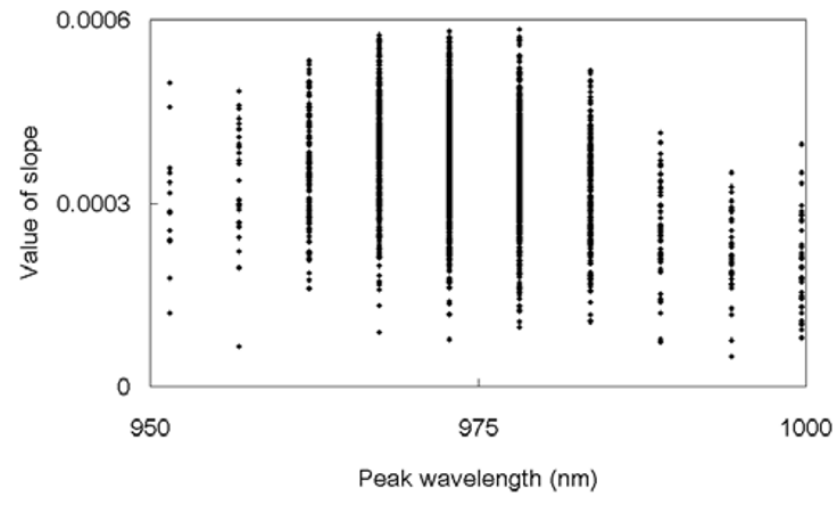

Fig. 7. Plot of peak wavelength versus value of slope of baseline at each pixel before spatial smoothing. The correlation coefficient between the value of slope of baseline and the peak position is -0.20 , indicating no correlation. The base data was obtained on 14 December.

crater (Lat: $15.4^{\circ} \mathrm{N}$, Long: $23.7^{\circ} \mathrm{E}$ )-Santos Dumont crater (Lat: $27.7^{\circ} \mathrm{N}$, Long: $4.8^{\circ} \mathrm{E}$ ). The line is shown in Fig. 1. One sigma for six maps is also shown in Fig. 8.

\section{Discussion}

In terms of this research, it is necessary to know whether or not the detected absorption-peak shifts indicate geological properties. Although random noise ( \pm approx. 5 counts before smoothing) is much smaller than the signal level (1500 2000 counts) of the lunar mare, the detection of peak position is still difficult because a change of only five counts of the pixel value might change the position of absorption peak of the ferroan iron. A statistical treatment is therefore required. We were able to extract the geological features of Mare Serenitatis from the smoothing processes and the comparison of the six absorption-peak maps (Fig. 5). Because these six absorption-peak maps of Mare Serenitatis (Fig. 5) were obtained under different conditions, such as the optical depth of the atmosphere and assigned CCD pixels, the area with common characteristics

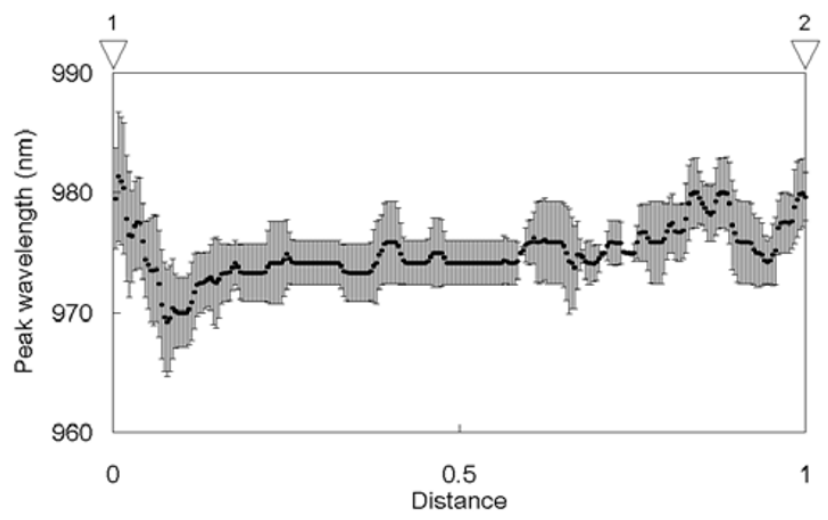

Fig. 8. Peak position profile derived from the line defined by two points, from the Plinius crater (Lat: $15.4^{\circ} \mathrm{N}$, Long: $23.7^{\circ} \mathrm{E}$ ) (left) to the Santos-Dumont crater (Lat: $27.7^{\circ} \mathrm{N}$, Long: $4.8^{\circ} \mathrm{E}$ ) (right). The line is shown in Fig. 1. The peak positions correspond to highland areas are ruled out. Peak positions are the average of all peak positions of six images (Fig. 5(a-f)). Error bar indicates one sigma of six images.

(areas 1 4 and perhaps 5 in Fig. 6) among these maps would indicate geological properties, not observation errors. Considering that one sigma of the peak positions of six images (Fig. 8) is $0-4 \mathrm{~nm}$ and that the spectral resolution of ALIS is about $5 \mathrm{~nm}$, most of the peak position would not change over a 5-nm scale on Fig. 5. The area where the standard deviation of peak position is wide may be the area where the peak position locates at the boundary of the spectral scale.

To estimate the geological properties of the characteristic area of our map, we divided the peak image derived from data set on 14 December into geological units based on Kodama and Yamaguchi (2003) (Fig. 9). Given that pyroxene is the most abundant mineral in the mare basalt (50$60 \mathrm{vol} \%)$, followed by plagioclase (20-40 vol\%), and that olivine is a relatively low abundant mineral $(<10 \mathrm{vol} \%)$ (BVSP, 1981) and knowing the laboratory spectral reflectance of these minerals (e.g., Pieters and Englert, 1993), we can conclude that the detected absorption-peak shifts would be caused mainly by the chemical variation of pyroxene. The band center of the highly diagnostic $1000 \mathrm{~nm}$ absorption band varies systematically between the band centers of the two components (orthopyroxene and clinopyroxene) and is a direct function of the mineralogy of the mixture. When orthopyroxene is clearly the dominant component, the band centers fall within a range from 900 to $930 \mathrm{~nm}$. When clinopyroxene is the dominant component, the band centers vary from 980 to $1000 \mathrm{~nm}$ (Pieters and Englert, 1993). The peak wavelengths of our map would not be the same as laboratory spectra; consequently, only a relative value could be used for our peak map because our mare spectra are normalized by the highland spectra and the definition of baseline is different from that of laboratory spectra. However, the composition of the Apollo samples suggests that the dominant mafic mineral in Mare Serenitatis is clinopyroxene (BVSP). Therefore, the subtle peak shifts we observed would be due to variations in clinopyroxene. The area where the peak position shifts to a longer wavelength than that of their surroundings may have a low Mg\#. Fac-

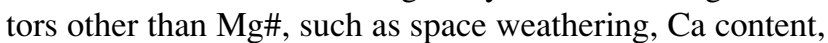






Fig. 9. The absorption-peak image with geological boundaries based on Kodama and Yamaguchi (2003). The base image was obtained on 14 December.

grain size, among other, are also able to shift the position of the absorption peak. However, we expect that $\mathrm{Mg \#}$ variation has been detected-at least in the same lava-because there is no correlation between the space weathering and the position of the peak (Fig. 7) and there would be correlation between $\mathrm{Ca}$ content and $\mathrm{Mg \#}$ in the same magma differentiation trend. To check whether the peak shift is caused by the change in chemical composition of the clinopyroxene of the mare basalt, the Mg\# map of Mare Serenitatis derived from Lunar Prospector Gamma Ray Spectrometer (LPGRS) (Prettyman et al., 2002) (Fig. 10(a)) was compared with our absorption peak map of Mare Serenitatis. Our map was resampled (Fig. 10(b)) to the similar spatial resolution to that of $\mathrm{Mg \#}$ map of LPGRS. The iron abundance map (Lawrence et al., 2002) of the same area as that of the $\mathrm{Mg} \#$ map and the $700 \mathrm{~nm}$ ALIS reflectance image of the same area as our absorption peak map are also shown in Fig. 10(c) and (d), respectively. The increase in Mg\# shifts the absorption peak to a shorter wavelength (e.g. Hazen et al., 1978). A comparison of Fig. 10(a) and (b) suggests that similar spatial patterns exist. The absorption peak shifts from lower to a higher wavelength (approximately corresponding to a decrease in $\mathrm{Mg \# )}$ in the area from the Mare Tranquillitatis to the center of Mare Serenitatis. However, the spatial resolution is too low to confirm the similarity between the Mg\# map of LPGRS and our peak-shift map.

On the other hand, there is a possibility that the lavas of areas 2 and 4 in Fig. 6 have a high Mg\#. The Sr5 lava unit, including areas 2 and 4, is the youngest within this mare according to Kodama and Yamaguchi (2003). Since the $\mathrm{TiO}_{2}$ concentration is almost constant throughout the Sr5 unit, all of the lava of this geological unit should have come from a common parent layer. Nevertheless, a peak shift is detected. If this peak shift suggests $\mathrm{Mg \#}$ variations, the variations lead two interpretations. One is that the variation is caused by the difference in the degree of partial melting of the parent rock. The lavas of high $\mathrm{Mg \#}$ areas, such as areas 2 and 4 in Fig. 6, may be at a higher degree of partial


Fig. 10. (a) Mg\# map of Mare Serenitatis derived from the Luna Prospector Gamma Ray Spectrometer (LPGRS) (Prettyman et al., 2002), (b) the absorption peak map of Mare Serenitatis obtained in this survey, (c) FeO abundance map of the same area as (a) derived from LPGRS (Lawrence et al., 2002), (d) the $700 \mathrm{~nm}$ reflectance image of the same area as (b) derived from ALIS. The data for (b) and (d) was obtained on 14 December. The absorption peak map (b) was resampled to the similar spatial resolution to that of $\mathrm{Mg} \#$ map of LPGRS.

melt in the latter stage. It could be interpreted that these are located near a vent. Another interpretation is that the variation is caused by the difference in the degree of magma differentiation after eruption. For a long flow, $\mathrm{Mg \#}$ would be expected to decrease along the flow line concomitant with the crystallization of the pyroxene and olivine with a higher Mg\# and their removal from the melt. In this case also, the high $\mathrm{Mg \#}$ area indicates the presence of a vent. Staid and Pieters (2001) reported another spectral variation along flows within the Imbrium basin; these researchers suggested variations in ilmenite content along previously mapped lava flows. However, such a trend could not be found in $\mathrm{TiO}_{2}$ map of areas 2 and 4 of the Mare Serenitatis. This may indicate variations in the fractionation pattern of mare basalt.

\section{Conclusion}

In conclusion: (1) the wavelength shift of the absorption spectra of ferrous iron at around $1000 \mathrm{~nm}$ was detected on Mare Serenitatis with the hyper-spectral telescope ALIS, (2) a map of the wavelength of the absorption peak on Mare Serenitatis was obtained, and (3) four notable areas with a short wavelength of absorption peak were detected, and there is a possibility that two areas among these, located in $\mathrm{Sr} 5$, indicate high $\mathrm{Mg} \#$ and, consequently, the presence of a vent. 
Acknowledgments. We would like to express our thanks to Dr. Mike Maberry and Mr. Daniel O'Gara of the University of Hawaii and the ALIS users group for their kind assistance at Haleakala site. We also wish to thank Drs. Shoichi Okano and Hiroaki Misawa of Tohoku University who allowed us to use their astronomical dome at the site and gave us helpful advice. We are grateful to Dr. Mark Robinson and an anonymous reviewer for helpful and constructive suggestions. ALIS was developed through Phase-IA research of "Ground-based Research Announcement for Space Utilization” (PI: K. Saiki) promoted by Japan Space Forum. Ground-based observations were supported by the Japanese Ministry of Education, Science, Sports, and Culture, Grant-in-Aid for Young Scientists (PI: K. Saiki).

\section{References}

Basaltic Volcanism Study Project (BVSP), Basaltic Volcanism on the terrestrial planets, 1286 pp, Pergamon Press Inc., New York, USA, 1981.

Blinder, A., Lunar Prospector: Overview, Science, 281, 1475-1476, 1998.

Boyce, M. J., Ages of flow units in the lunar nearside maria based on Lunar Orbiter IV photographs, Proc. Lunar Planet. Sci. Conf., 7, 2717-2728, 1976.

Carr, M. H., Geologic map of the Mare Serenitatis region of the moon, Geological survey, Map I-489, 1966.

Hazen, R. M., P. M. Bell, and H. K. Mao, Effects of compositional variation on absorption spectra of lunar pyroxenes, Proc. Lunar Planet. Sci. Conf., 9, 2914-2934, 1978.

Howard, K. A., M. H. Carr, and W. R. Muehlberger, Basalt stratigraphy of southern Mare Serenitatis, Apollo 17 preliminary science report, Washington D.C., U.S. Government Printing Office, NASA SP-330, 291-29-12, 1973

Kodama, S. and Y. Yamaguchi, Lunar mare volcanism in the eastern nearside region derived from Clementine UV/VIS data, Meteor. Planet. Sci., 38, 1461-1484, 2003.

Lawrence, D. J., W. C. Feldman, R. C. Elphic, R. C. Little, T. H. Prettyman, S. Maurice, P. G. Lucey, and A. B. Binder, Iron abundances on the lunar surface as measured by the Lunar Prospector GammaRay and Neutron Spectrometers, J. Geophys. Res., 107(E12), 5130, doi:10.1029/2001JE001530, 2002.

Lucey, P. G., G. J. Taylor, and E. Maralet, Abundance and distribution of iron on the Moon, Science, 268, 1150-1153, 1995.

Lucey, P. G., D. T. Blewett, and B. R. Hawke, Mapping FeO and $\mathrm{TiO}_{2}$ content of the lunar surface with multi-spectral imagery, J. Geophys. Res., 103, 3679-3699, 1998.

Lucey, P. G., D. T. Blewett, and B. L. Jolliff, Lunar iron and titanium abundance algorithms based on final processing of Clementine ultravioletvisible images, J. Geophys. Res., 105, 20297-20305, 2000.

Nozette, S., P. Rustan, L. P. Pleasance, D. M. Horan, P. Regeon, E. M. Shoemaker, P. D. Spudis, C. H. Acton, D. N. Baker, J. E. Blamont, B. J. Buratti, M. P. Corson, M. E. Davies, T. C. Duxbury, E. M. Eliason, B. M. Jakosky, and J. F. Kordas, The Clementine mission to the Moon: Scientific overview, Science, 266, 1835-1839, 1994.

Pieters, C. M., Mare basalt types on the front side of the Moon: A summary of spectral reflectance data, Lunar Planet. Sci. Conf., 9, 2825-2849, 1978.

Pieters, C. M. and A. J. Englert, Remote Geochemical Analysis: Elemental and Mineralogical Composition, Cambridge, 594pp, The Press Syndicate of the University of Cambridge, Cambridge CB2 2RU, United Kingdom, 1993.

Prettyman, T. H., W. C. Feldman, D. J. Lawrence, G. W. McKinney, A. B. Binder, R. C. Elphic, O. M. Gasnault, S. Maurice, and K. R. Moore, Library least squares analysis of Lunar Prospector gamma-ray spectra, 33rd Lunar Planet. Sci. Conf., Abstract \#2012, 2002.

Saiki, K., R. Nakamura, F. Ichikawa, H. Akiyama, and H. Takeda, Development of a telescope imaging spectrometer for the moon, Lunar Planet. Sci. Conf., XXXV \#148, 2004.

Sasaki, S., K. Nakamura, Y. Hamabe, E. Kurahashi, and T. Hiroi, Production of iron nanoparticles by laser irradiation in a simulation of lunarlike space weathering, Nature, 410, 555-557, 2001.

Scheaffer, G. A. and O. A. Scheaffer, ${ }^{39} \mathrm{Ar}-{ }^{40} \mathrm{Ar}$ ages of lunar rocks, Lunar Planet. Sci. Conf., 8, 2253-2300, 1977.

Staid, M. I. and C. M. Pieters, Mineralogy of the last lunar basalts: Results from Clementine, J. Geophys. Res, 106(E11), 27,887-27,900, 2001.

Stolper, E., Experimental petrology of eucritic meteorites, Geochem. Cosmochim., 41, 587-611, 1977.

Tera, F., D. A. Papanastassiou, and G. J. Wasseburg, Isotopic evidence for a terminal lunar cataclysm, Earth Planet. Sci. Lett., 22, 1-21, 1974.

Wilhelms, D. E. and F. M. McCauley, Geologic map of the nearside of the Moon, U. S. Geological Survey, Map I-703, Washington D.C., 1971.

H. Okuno (e-mail: hokuno@astroboy.ess.sci.osaka-u.ac.jp), Y. Yamanoi, and K. Saiki 